\title{
Robust phase estimation for signals with a low signal-to-noise-ratio
}

U. von Toussaint

Citation: AIP Conference Proceedings 1641, 246 (2015); doi: 10.1063/1.4905985

View online: http://dx.doi.org/10.1063/1.4905985

View Table of Contents: http://aip.scitation.org/toc/apc/1641/1

Published by the American Institute of Physics 


\title{
Robust phase estimation for signals with a low signal-to-noise-ratio
}

\author{
U. von Toussaint \\ Max-Planck-Institute for Plasma Physics, Boltzmannstrasse 2, 85748 Garching, Germany
}

\begin{abstract}
The estimation of time-dependent phase-shifts of distorted signals is an ubiquitous problem in signal-processing. Processing of data from plasma interferometry reveals that the standard approach based on band-pass filtering can result in misleading results already for moderate signal-to-noise ratios. An alternative phase estimation method based on a combination of direct signal matching and a directional statistics based Kalman filter is shown to yield superior phase-shift estimations for a wide range of conditions. The approach is illustrated on selected data and possible further enhancements are outlined.
\end{abstract}

Keywords: Phase estimation, Directional statistics, Kalman filter

PACS: $02.50 . \mathrm{Tt}, 02.50 . \mathrm{Bf}, 07.60 . \mathrm{Ly}$

\section{INTRODUCTION}

Estimation of phase-shifts is an essential part of spectral analysis [1]. In addition most interferometry based diagnostics (eg. Mach-Zehnder interferometer [2], synthetic aperture radar [3], speckle-measurements [4]) yield data where the total phase shift $\varphi_{t}$ is not directly accessible but wrapped into $\varphi \in[-\pi ; \pi]$. Due to the ubiquitous occurrence of phasewrapping [5] many phase unwrapping methods based on different principles have been developed $([6,7,8,9])$ but the problem remains difficult and under active investigation. Here we focus on the estimation of phase-shifts based on 1-D time-series data from a Mach-Zehnder-type interferometer used for electron density measurements in high-temperature plasma experiments. Although in some respects less demanding than the phase-estimation of two-dimensional data sets like InSAR images[10], the signal properties with correlated outliers and superimposed distortions with timevarying frequencies (eg. MHD-modes) still challenge standard approaches. Here we present an approach consisting of a combination of direct shift estimation and a non-standard Kalman smoothing filter as an alternative for the phase estimation problem.

\section{BACKGROUND}

\section{Principles of plasma interferometry}

The interaction of a plasma and an electromagnetic wave is described by Maxwell's equations. Under the assumption of quasi-neutrality of the plasma, quasi-static ions and within the linear-response regime the plasma-frequency $\omega_{p}$ is a function of the electron-density $n_{e}$,

$$
\omega_{p}=\sqrt{\frac{e^{2} n_{e}}{\varepsilon_{0} \mathrm{~m}_{e}}}
$$

with electron mass $\mathrm{m}_{e}$, elementary charge $e$ and the permittivity of free space $\varepsilon_{0}=8.8542 \times 10^{-12} \mathrm{~m}^{-1} \mathrm{~F}$. The phase velocity of an electromagnetic wave with angular frequency $\omega$ and wave vector $\vec{k}$ in a plasma can then be expressed as

$$
v_{p h}=\frac{\omega}{k}=\frac{c}{\sqrt{1-\frac{\omega_{p}^{2}}{\omega^{2}}}}
$$

which yields the refractive index of the plasma $n$ as

$$
n=\frac{c}{v_{p h}}=\sqrt{1-\frac{\omega_{p}^{2}}{\omega^{2}}}
$$

Bayesian Inference and Maximum Entropy Methods in Science and Engineering (MaxEnt 2014)

AIP Conf. Proc. 1641, 246-254 (2015); doi: 10.1063/1.4905985

(C) 2015 AIP Publishing LLC 978-0-7354-1280-4/\$30.00 
It is noteworthy that the refractive index $n$ of a plasma is less than 1 , unlike most other optical media. The phase-shift of a laser beam passing through a plasma with respect to a reference beam depends on the difference of the optical path lengths. The optical path length of the beam penetrating the plasma is given by

$$
L_{\text {opt }}=\int_{0}^{L_{\text {geom }}} \mathrm{d} x \frac{c}{v_{p h}}=\int_{0}^{L_{\text {geom }}} \mathrm{d} x \sqrt{1-\frac{e^{2} n_{e}}{\varepsilon_{0} \mathrm{~m}_{e} \omega^{2}}}
$$

As long as the angular beam frequency $\omega$ is much higher than the plasma frequency $\omega_{p}$ the square root can be expanded in a Taylor series $(\sqrt{1-x}=1-x / 2+\ldots)$, simplifying the expression to

$$
L_{o p t}=\int_{0}^{L_{g e o m}} \mathrm{~d} x\left(1-n_{e}(x) \frac{e^{2}}{2 \varepsilon_{0} \mathrm{~m}_{e} \omega^{2}}\right)
$$

which can be rewritten (introducing the vacuum wavelength $\lambda_{0}=2 \pi c / \omega$ of the laser and the classical electron radius $\left.r_{e}\right)$ as

$$
L_{\text {opt }}=L_{\text {geom }}-\frac{r_{e} \lambda_{0}^{2}}{2 \pi} \int_{0}^{L_{g e o m}} \mathrm{~d} x n_{e}(x)
$$

The phase difference of the reference beam and the beam propagating through the plasma is then a function of the plasma electron density only

$$
\varphi=\lambda_{0} r_{e} \int_{0}^{L_{\text {geom }}} \mathrm{d} x n_{e}(x)
$$

thus providing a direct measure of the integrated plasma electron density along the propagation line of the beam.

\section{Experimental Set-Up}

The plasma induced phase shift is in many magnetic fusion experiments measured by Mach-Zehnder interferometers. A typical set-up is given in Figure1 [11]. A laser beam is splitted into three beams. One beam passes through the plasma, the second beam is used as reference signal. However, the frequencies used are in the order of $10^{13} \mathrm{~Hz}$ and cannot be resolved. Therefore the frequency of the third beam is Doppler shifted (cf. Eq. 10) by a tiny amount of $\Delta \omega \approx 10^{4} \mathrm{~Hz}$, thus providing a beat signal after superposition with the first two beams according to

$$
\sin (\omega t)+\sin ((\omega+\Delta \omega) t)=2 \sin \left(\frac{2 \omega+\Delta \omega}{2} t\right) \cos \left(\frac{\Delta \omega}{2} t\right) .
$$

The fast optical frequency $\omega$ is not resolved by the detector, resulting in a signal varying with the beat frequency $\Delta \omega$ (please note that the square of the amplitude is the measured signal). The same considerations hold for the superposition of the beam passing through the plasma and the frequency shifted beam:

$$
\sin (\omega t+\varphi)+\sin ((\omega+\Delta \omega) t)=2 \sin \left(\frac{2 \omega+\Delta \omega}{2} t+\frac{\varphi}{2}\right) \cos \left(\frac{\Delta \omega}{2} t-\frac{\varphi}{2}\right) .
$$

A comparison of the phase of the two beat signals allows to extract information on the line integrated density of the plasma. The actual equations used are slightly more complicated because the different amplitudes of the beams (eg. due to attenuation, stray light, mirror reflectivity) need to be taken into account[12].

The required frequency shift is accomplished by reflecting the beam from a rotating grating. The frequency of the reflected beam is Doppler shifted and is given by

$$
\Delta \omega=\omega\left(\sqrt{\frac{c+2 v}{c-2 v}}-1\right)
$$

where $v$ denotes the speed of the grating with respect to the beam direction and $c$ is the velocity of light. Due to the hostile environment (strong and fluctuating magnetic and electrical fields) in tokamak experiments, the grating is mounted on a cylinder (see upper left part of Figure 1) which is driven by pressurized air. 


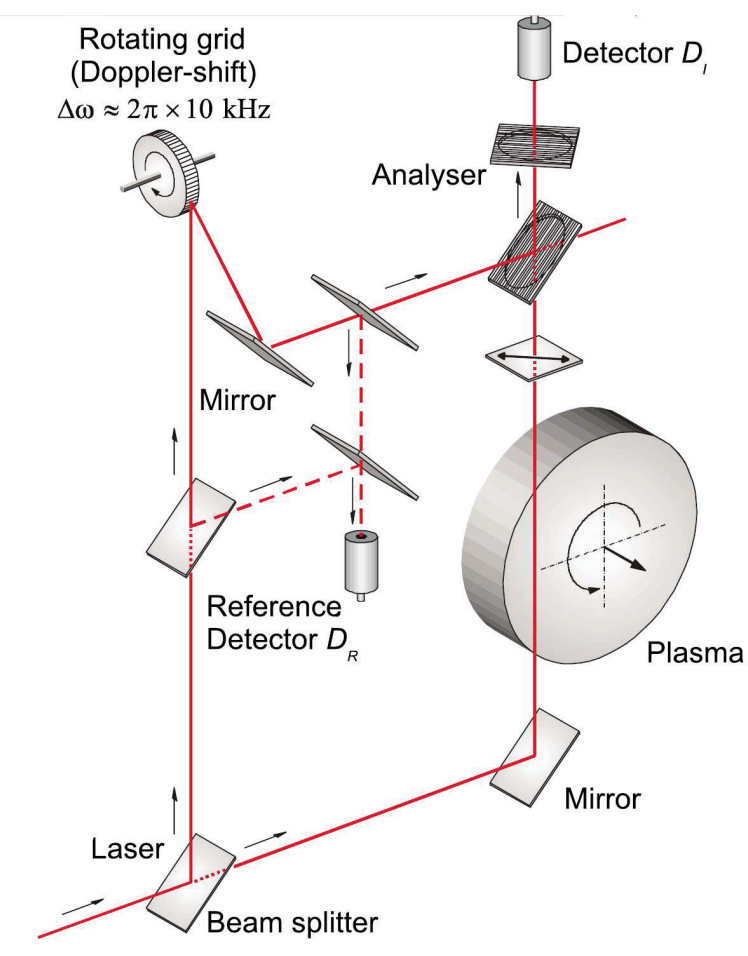

FIGURE 1. Simplified schematic of Mach-Zehnder interferometer set-ups for plasma density measurements at tokamak devices (adapted from [11])
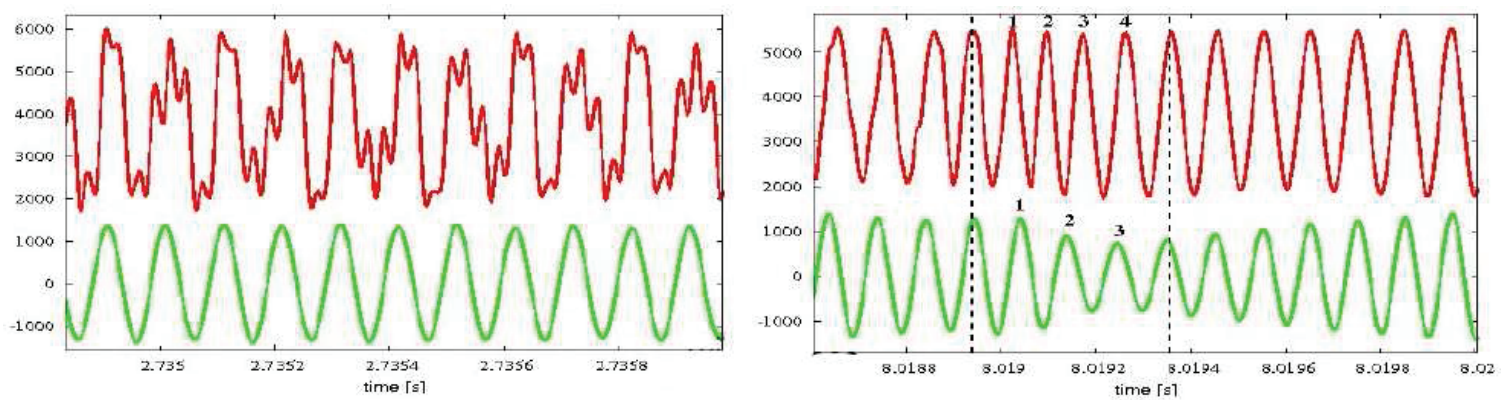

FIGURE 2. Effect of signal-filtering with bandpass of $9.5 \mathrm{kHz}-10.5 \mathrm{kHz}$ on signal. The upper time traces display the raw signal, the lower time traces provide the signal after band-pass filtering. Although in many instances the signal quality is improved considerably, fringes may be lost in other situations without an obvious triggering event (cf. different number of amplitude maxima in the raw and band-pass filtered signal displayed in the right hand panel).

\section{Signal processing}

For concreteness we describe the signal processing presently applied at ASDEX Upgrade [13] only. However, the general methodology is very similar at most tokamak and stellarator devices [14]. The detector signals at the tokamak experiment ASDEX Upgrade are digitized using analog-to-digital converters (ADC) with a sampling rate of $1 \mathrm{MHz}$ with 14 bit resolution, providing an oversampling factor of 100 of the beat-wave signal. For physics reasons (beam deflection, sensitivity) the applied laser wavelengths are in the range of around 100-300 $\mu \mathrm{m}$, at ASDEX Upgrade a deuterium-cyanide (DCN) laser with a wavelength of $195 \mu \mathrm{m}$ is used. For the phase reconstruction the signal is bandpass filtered with the center frequency set to the beat frequency $\Delta \omega$ and a bandpass-width of $1 \mathrm{kHz}$. Then the signal is 
divided into equidistant segments, each of them containing at least one full period. The function

$$
h(t \mid A, B, \varphi)=A \sin \left(\omega_{A} t+\varphi\right)+B
$$

is then fitted to each segment and the parameters $A, B, \varphi$ are derived from each segment. It should be pointed out that there are several local minima and that the optimization is very sensitive to the choice of the initial parameter values. Phase wraps are identified by the assumption that phase changes are always less than $\pi$ between to segments.

For many plasma scenarios this algorithm yields fits with a low residuum - in part due to the removal of other signal contributions with frequency components different from the beat frequency. This can be seen on the left hand of Figure 2, where some superimposed high frequency component is filtered. One drawback of the band-pass filter is displayed in the right panel of Figure 2: Although the raw signal exhibits a pronounced sinusoidal structure (although with slightly changing cycle length), the band-pass filter removes a full cycle, thus resulting in a spurious jump in the derived plasma density. The manual removal of these spurious events is very tiring, error prone and cannot be afforded for the next generation of experiments where the plasma discharges are in the order of 30 minutes and/or the plasma density is adjusted by automated feedback-control schemes.

\section{SIGNAL PROPERTIES}

The most prominent perturbations of the signal in tokamak experiments can be divided into three categories [15]

- Magneto-hydrodynamic modes (MHD)

- Edge-localized modes (ELMs)

- Pellet injections.

The description of the underlying physics of these phenomena is beyond the scope of the present paper, but the resulting distortions of the signal have a characteristic fingerprint which we want address briefly. MHD modes are distortions of the plasma symmetry (eg. rotating islands) where temperature or density differ from the surrounding plasma. In effect the beat signal experiences amplitude modulations at the MHD mode frequency $f_{\text {MHD }}$ resulting in a detector signal with additional frequency components $f_{\text {Beat }}+f_{\text {MHD }}$ and $\left|f_{\text {Beat }}-f_{\text {MHD }}\right|$ besides the beat frequency $f_{\text {Beat }}$.

ELMs are very short $(\Delta t<0.5 \mathrm{~ms})$ distortions of the plasma edge, resulting in a sudden decrease of the plasma density and high power loads on the plasma-facing components followed by a recovery phase until the next ELM event happens. The typical signature of ELMs is a short dip in the signal intensity, presumably caused by beam diffraction on the high-density filaments of ELMs [15].

As means of plasma and density control cryogenic deuterium pellets are injected into the plasma. The evaporation of the pellets results in a fast density increase, accompanied by a rapid phase change. While for small and medium sized pellets the phase shift is typically below $\pi$, larger pellets may lead to changes exceeding that value. In the latter case the typical signature of pellets and available prior information on the size of the pellet to be injected could be utilized to analyze also these data - although the phase data may be insufficient on their own.

Based on these main perturbations test signals were developed which exhibit the key features of the distortions. The test signal is sampled at $1 \mathrm{MHz}$, has on average a relatively constant amplitude (see upper panel of Figure 3 with point-wise independent Gaussian noise with mean zero superimposed. It reflects in parts a quiescent plasma discharge (second panel from the top), where the phase of the probe signal (solid line) shifts only slowly with respect to the reference signal (dashed line). In other parts of the signal short dips in the signal are present (see middle/right part of the third panel of Figure 3) as well as signal components emulating MHD modes with frequency sweeps with an amplitude of up to $1 / 3$ of the undistorted beat-wave signal (cf. signal-shape on the left hand side of the third panel in Figure 3). The lower panel displays the 'true' phase-shift between the probe beam and the reference beam underlying the test-signal, wrapped into the interval $[-\pi ; \pi[$.

\section{SIGNAL ANALYSIS}

Based on the signal characteristic we tried different approaches to estimate the phase-shifts

- Kalman-filter based estimation of the measured signals

- Estimation of the phase-shift induced frequency shifts 


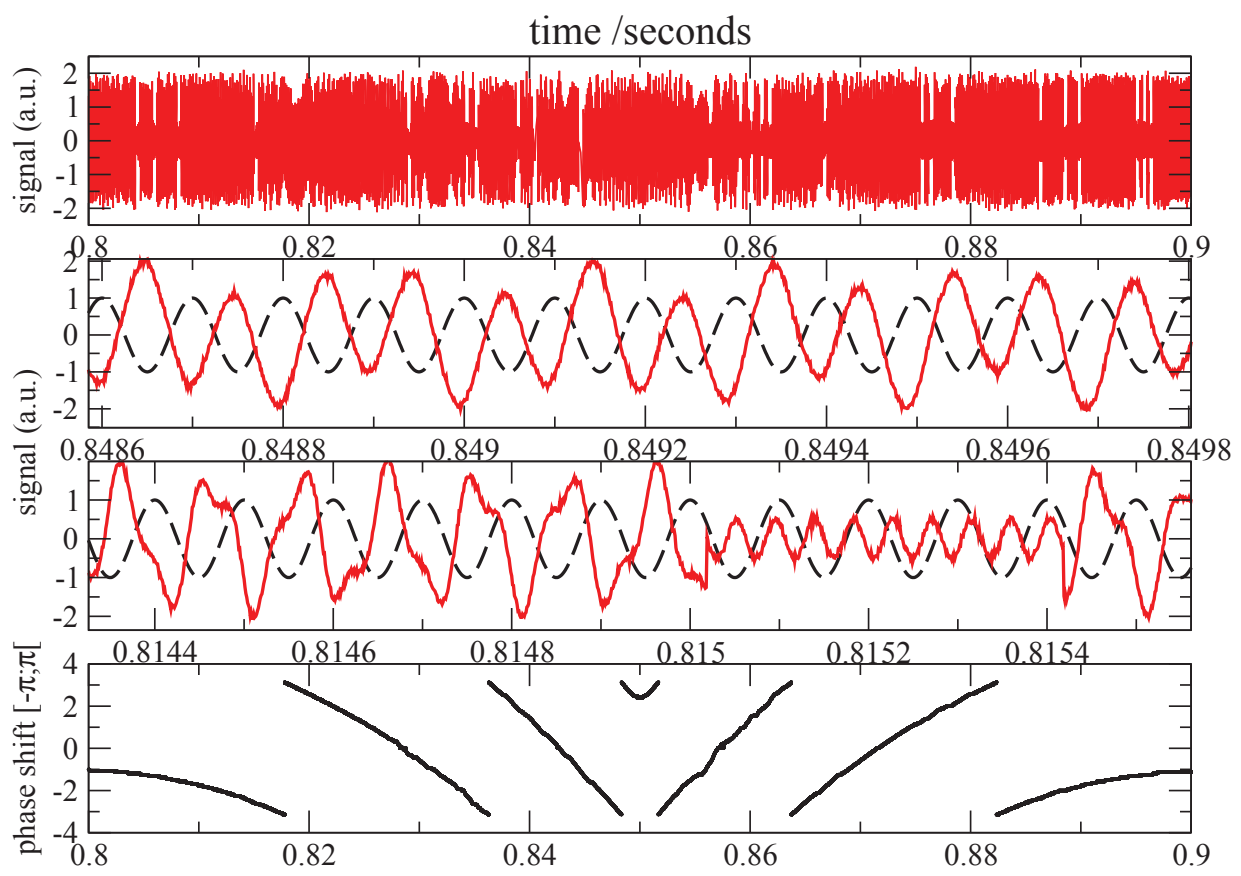

FIGURE 3. Mock data exhibiting the typical features of the measured signals with signal outages (due to ELMs or pellet injection, significant contributions in other frequency bands and phase shifts of varying rate. The lower panel displays the 'true' phase-shift between the probe beam and the reference beam underlying the test-signal, wrapped into the interval $[-\pi ; \pi[$.

- Correlation estimation with subsequent phase unwrapping

which are detailed below.

\section{Kalman filter}

A Kalman filter [16] is essentially an efficient way to estimate the state space of a probabilistic state space model, where the system dynamics (change from the state $\underline{x}_{k-1}$ at instance $k-1$ to the state $\underline{x}_{k}$ at instance $k$ ) is given by [17]

$$
\underline{x}_{k} \sim p\left(\underline{x}_{k} \mid \underline{x}_{k-1}\right) \underline{y}_{k} \sim p\left(\underline{y}_{k} \mid \underline{x}_{k}\right) \text { for } \mathrm{k}=1,2, \ldots, \text { where }
$$

- $\underline{x}_{k}$ is the state of the system at time step $k$

- $\underline{y}_{k}$ is the measurement at time step $k$,

- $p\left(\underline{x}_{k} \mid \underline{x}_{k-1}\right)$ is the dynamic model which describes the evolution of the probability density of the state space,

- $p\left(\underline{y}_{k} \mid \underline{x}_{k}\right)$ is the measurement model, describing the probability of the measurement $\underline{y}_{k}$ if the model is in state

We applied an extended Kalman Filter (EKF) to the state space $\underline{x}=A, B, \varphi$ with $p\left(\underline{x}_{k} \mid \underline{x}_{k-1}\right)$ distributed as a multivariate Gaussian distribution with mean zero and a diagonal covariance matrix. The mapping form the state space $\underline{x}$ to the observed (scalar) signal $y$ was given by Eq.11, again with a Gaussian distribution for the measurement error.

The experience with this approach were mixed. Although the extended Kalman filter is extremely fast and allows for real-time analysis, it turned out to be difficult to assign the parameters of the state space covariance matrix in a robust manner. If the covariance was chosen too large, the magnitude of the phase values sometimes just grew too fast, thus allowing a perfect fit of the data (residual was approaching zero) and the filter never recovered for the rest of 
the data set. A too restrictive setting, on the other hand, just could not follow the fast changes due to ELMs or MHD modes. It may well be that a more refined parameter tuning or the use of switching state space Kalman filters could yield a more robust algorithm - but the number of input parameters which need to be adjusted in these situations were discouraging and we looked for other approaches.

\section{Frequency estimation}

The next attempt was based on the insight that a change of the phase is equivalent to a frequency change, or more precisely, that (to first order) the time derivative of the phase signal is identical to a frequency offset:

$$
\Delta f(t)=\frac{\mathrm{d} \varphi(t)}{\mathrm{d} t}
$$

Therefore the time-integrated difference of the local frequency of the probe signal and the reference signal yields the total phase difference. This approach has a big advantage: Unlike most other approaches it is not affected by the phase wrapping problem, which often invalidates all subsequent data points if there has been a single wrong assignment. In addition the integration over time is intrinsically smoothing the signal noise and thus provides an algorithm which is stable against individual outliers. It is tempting to use a windowed FFT based algorithm (eg. Lomb-Scargle periodigram [18]) to evaluate the local frequency. However, as has been shown by Bretthorst [19] the application of FFT for frequency estimation is inferior if (besides noise) more than one frequency is present in the signal. Therefore we implemented the algorithm of Bretthorst [19], which computes an orthonormal basis on the support of the data set (which need not to be equidistant) and estimates the probability (unlike FFT) for the presence of a given frequency or frequency doublets or higher order multiplets. A typical result is displayed in the upper panel of Figure 4, where we analyzed the signal provided in Figure 3. The integration of the difference in frequency between the reference signal with $10 \mathrm{kHz}$ and the test signal yields the phase shift plotted in the lower panel of Figure 4 (please note that a higher frequency of the test signal compared to the reference signal results in a negative phase shift). It matches the true phase shift quite closely, except at $t=0.855$ where a small spurious kink appears.

Although the performance on the test data sets was quite reasonable, application of the algorithm to measured data sets revealed an unexpected problem: Also the frequency of the reference beam shows significant frequency fluctuations with contributions scattered over the whole range of the spectrum. Apparently the drive of the rotating grid with pressurized air is less stable than expected. In addition there are some high frequency components (multiple of $10 \mathrm{kHz}$ ) which are presumable due to lamella entering or leaving the air stream. These frequency fluctuations in the reference signal require that the phase shift due to the plasma is computed via the (integration of the) difference of two estimated frequencies. This accumulates significant uncertainties over time - turning one of the advantages of this approach (signal integration) into a disadvantage. The results indicate that the frequency based approach is in principle feasible and could be valuable as an alternative way to process the data. However, to exploit its potential the most important step would be a better frequency stability of the reference beam, eg. by generating the beat frequency using an acusto-optical modulator instead of a pressurized air driven rotating cylinder.

\section{Direct phase-shift estimation}

Based on the insight that both beams, reference and probe beam show noticeable frequency fluctuations over time (thus excluding all 'global' approaches), the idea of a direct local estimation of the (wrapped) phase shift appeared most promising, where the problem of the total phase-shift estimation is shifted to a post-processing step.

In a first step the local autocorrelation time (ie. shift in number of samples) of the reference beam is computed, where the resolution is given by the oversampling factor, the ratio of beat frequency and sample frequency, ie. $\approx 0.01$ at ASDEX Upgrade. The cross-correlation of probe and reference beams is computed based on this autocorrelation length. The result for the test-data set is shown in Figure 5, where the magnitude of the cross-correlation is represented by the various levels of gray.

The result of this cross-correlation computation is promising - but it has a severe drawback: The associated probability distribution for the computed cross-correlation values is unknown, ie. how much more likely is a crosscorrelation value of 0.9 compared to a value of 0.8 ? This does not allow for further processing steps relying on the uncertainty of the phase-shift estimate. A different measure is needed. 


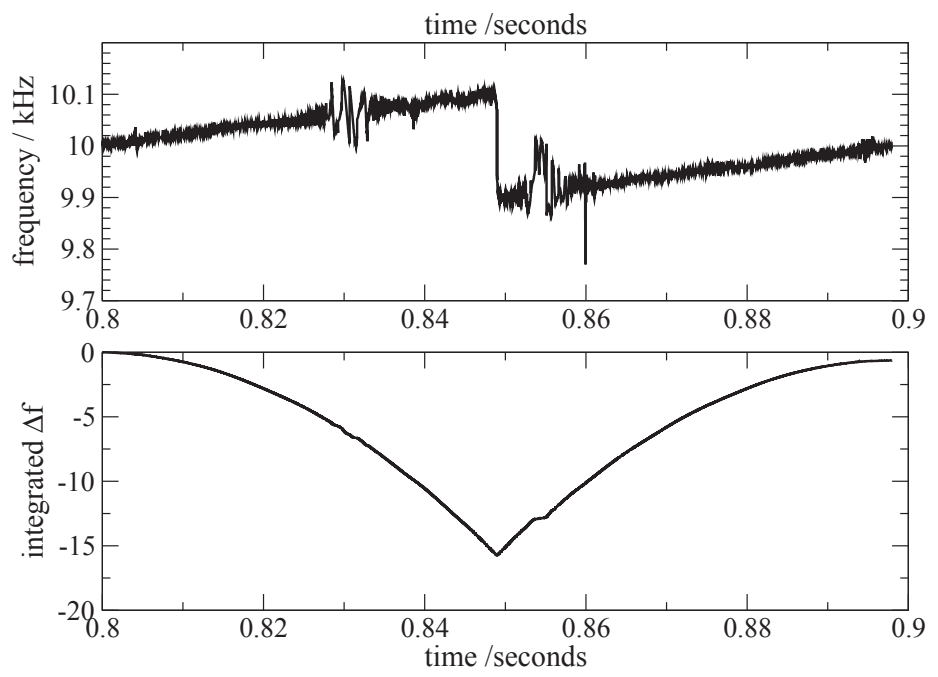

FIGURE 4. Phase estimation based on the test data displayed in Figure 3. Upper panel: Estimated time-dependent frequency of the test signal reflecting the change of the signal phase. The reference frequency is $f=10 \mathrm{kHz}$. Lower panel: The integrated difference of the reference frequency to the test signal frequency provides a robust measure of the total phase difference for most of the time, but is disturbed if side band signals become to strong in intensity.

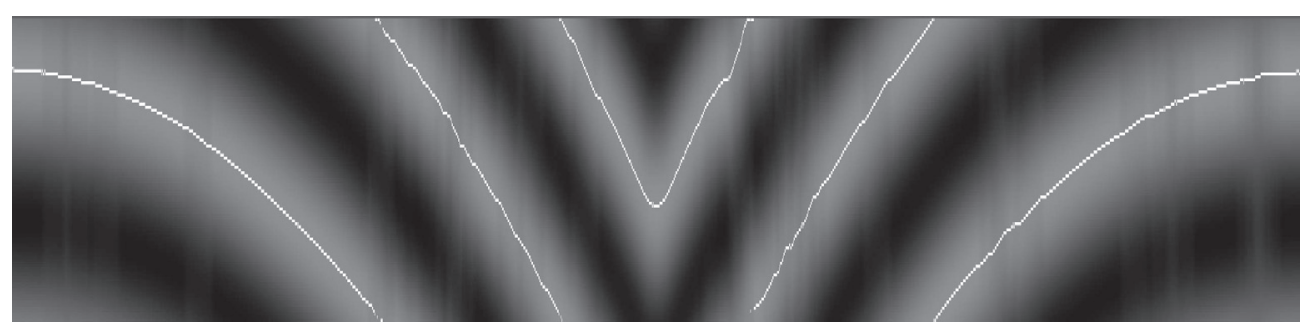

FIGURE 5. Cross correlation of the reference and the test signal. The time axis is the abscissae, the phase shift between $[-\pi ; \pi[$ is on the ordinate and the strength of the cross-correlation is given as gray level. The maximum value for for each time-step is indicated by a white spot. The overall appearance is smooth, without severe outliers of the positions of the maximum of the cross-correlation.

We derive the probability of the phase-shift $\tau_{i}$ between the reference-beam and the probe-beam by considering the probe beam as noisy and shifted version of the reference beam

$$
\Delta d\left(t \mid \tau_{i}\right)=d_{\text {ref }}(t)-d_{\text {probe }}\left(t+\tau_{i}\right) .
$$

The probability for a phase-shift of $\tau_{i}$ is computed based on the Laplace-norm (to increase stability against individual outliers) of the difference-signal, normalized by the complete set of values calculated for all (discrete) phase-shifts between zero and the autocorrelation length. This yields the probability for the wrapped phase-shifts as function of time, which, however, still needs to be unwrapped.

Here we suggest the following: The appropriate space for the data is a (unit) cylinder surface $(t, \varphi)$ in $3 \mathrm{~d}$ Euclidean space, where the cylinder axis is given by time and corresponds to $z$-axis and the phase angle provides the position on the cylinder surface via $x=\cos (\varphi), y=\sin (\varphi)$. In this space no phase wrapping occurs - it is only a consequence of the information loss by mapping the data into either the x-z-plane or y-z-plane. This mapping needs to be reflected in the probabilistic description of the signal. In addition the uncertainty in the phase has to reflect the $2 \pi$-periodicity of the signal also. It is noteworthy that often the von-Mises distribution is used for probability distributions on the circle, but in our case (Gaussian uncertainty) the appropriate distribution is given by the wrapped Normal distribution $p_{W N}(\varphi \mid \mu, \sigma)[20]$, which is essentially provided by wrapping the Gaussian distribution around the unit circle, thus 
ensuring the $2 \pi$ - periodicity:

$$
p_{W N}(\varphi \mid \mu, \sigma)=\frac{1}{\sigma \sqrt{2 \pi}} \sum_{k=-\infty}^{\infty} \exp \left[\frac{-(\varphi-\mu+2 \pi k)^{2}}{2 \sigma^{2}}\right] .
$$

What remains to be done is to find an efficient way to solve the Chapman-Kolmogorov-equation

$$
p\left(\varphi_{k} \mid \tau_{1: k-1}\right)=\int d \varphi_{k-1} p\left(\varphi_{k} \mid \varphi_{k-1}\right) p\left(\varphi_{k-1} \mid \tau_{1: k-1}\right)
$$

and the update to $p\left(\varphi_{k} \mid \tau_{1: k}\right)$ based on measurement $\tau_{k}$. Here we employ for an approximate solution a RauchTung-Striebel smoother based on the quite recently developed wrapped Kalman filter [21]. This post-processing for unwrapping is very efficient and requires only seconds for more than $10^{5}$ measurements and has turned out to be quite robust. The reference signal after unwrapping is shown in Figure 6. The turning point as well as the changing height of the windings is clearly visible, The application of this two-step algorithm has provided quite robust and reliable

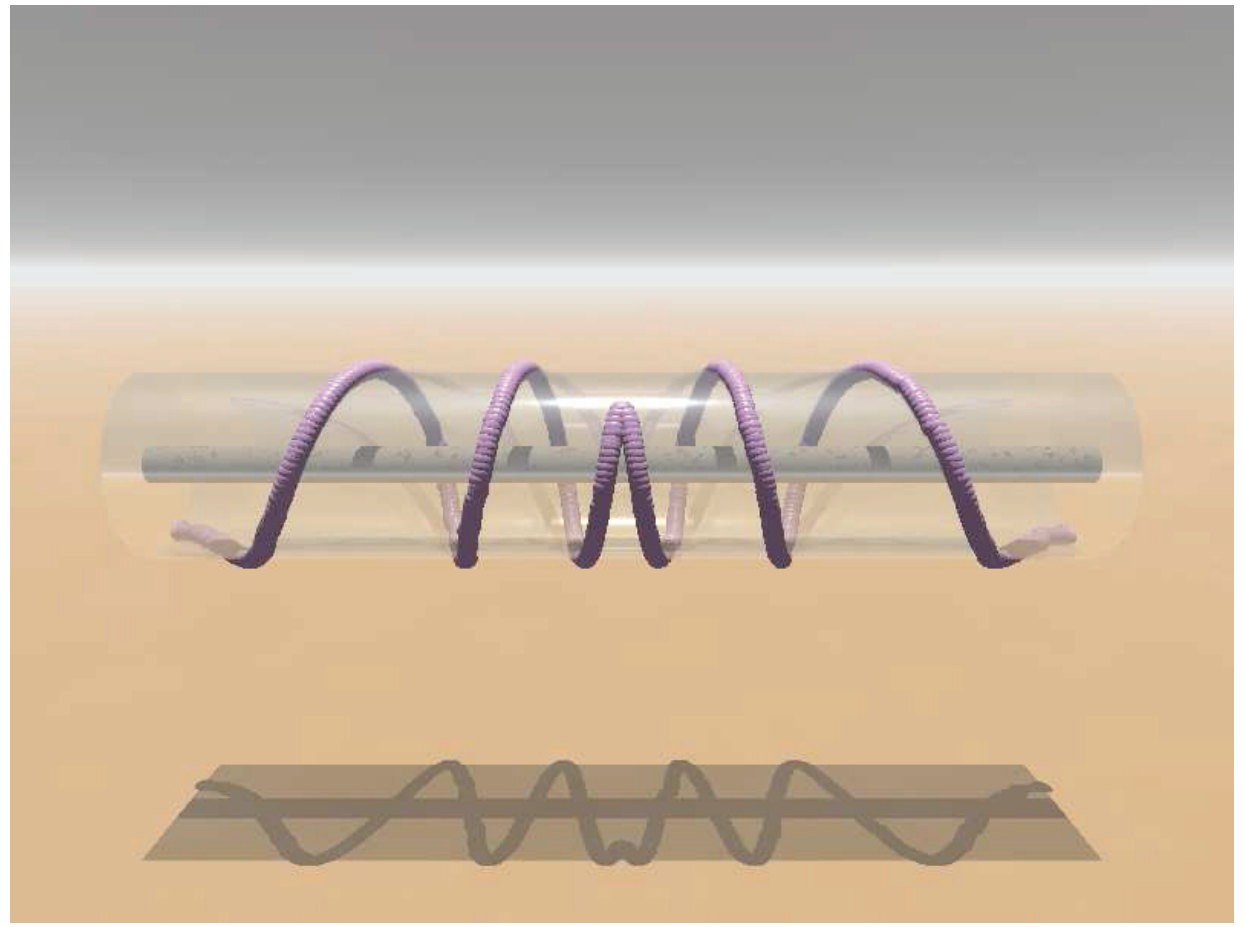

FIGURE 6. Unwrapped phase shift mapped onto $S^{1} \times R$-space (phase angle and time) together with the phase values used to generate the test data. The latter values are not visible because the difference between these and the inferred phase value is too small.

results on mock data as well as on all of the selected measurement data. Now a comprehensive test with the shot-data base at ASDEX Upgrade is planned as next step.

\section{CONCLUSION AND OUTLOOK}

We have tested several approaches to the problem of phase estimation in time-series data. While the standard approach (using Kalman filters for phase tracking has shown to be quite sensitive to the characteristics of the data, two other approaches yielded better results. The phase estimation based on integrated frequency differences appeared promising, but is (in the investigated experimental set-up) hampered by frequency fluctuation of the reference beam. Nevertheless, it could be useful as an alternative approach for phase-estimation, eg. to achieve a higher robustness for controlpurposes. The computational effort, however, is high. 
The most promising approach uses a two-step algorithm. In a first step the probability distribution for the phaseshift is computed, followed by a RTS-smoother using the wrapped Gaussian distribution for the data-likelihood. The experience so far (based on mock and measurement data) is excellent. In a next step the algorithm will be applied to the shot-data base of ASDEX Upgrade for a comprehensive characterization.

\section{ACKNOWLEDGMENTS}

We are very grateful to A. Mlynek who brought this problem to our attention. He not only assisted with his profound knowledge about the interferometry set-up and associated data acquisition system but who also provided test examples challenging the various approaches.

\section{REFERENCES}

1. S. Marple, Digital Spectrum Analysis with Applications, Prentice-Hall, New Jersey, 1987.

2. H. Soltwisch, Review of Scientific Instruments 57, 1939-1944 (1986).

3. M. Skolnik, Radar Handbook, 3rd edition, McGraw-Hill, New York, 2008.

4. U. von Toussaint, S. Gori, and V. Dose, Applied Optics 43, 5356-5363 (2004).

5. K. Itoh, Applied Optics 21, 2470 (1982).

6. M. Pritt, IEEE Trans. Geosci. Remote Sens. 34, 728-738 (1996).

7. T. Flynn, J. Opt. Soc. Am. A 14, 2692-2701 (1997).

8. E. Berger, W. von der Linden, V. Dose, M. Jakobi, and A. Koch, Applied Optics 38, 4997 (1999).

9. C. W. Chen, and H. A. Zebker, JOSA A 17, 401-414 (2000).

10. D. Ghiglia, and M. Pritt, Two-dimensional Phase Unwrapping: Theory, Algorithms and Software, John-Wiley and Sons, New York, 1998.

11. G. Bertschinger, C. Endres, F. Lewen, and J. Oosterbeek, Review of Scientific Instruments 79, 10E709 (2008).

12. A. Mlynek, Real-time control of the plasma density profile on ASDEX Upgrade, Ph.D. thesis, Ludwig-Maximilians-Universität München (2010).

13. Improved phase detection schemes for plasma interferometry,P6.012, 40th EPS conference on Plasma Physics, Espoo, Finland, European Physical Society, 2013.

14. C. Gil, A. Barbuti, D. Elbeze, P. Pastor, J. Philip, and L. Toulouse, Review of Scientific Instruments 79, 10E710 (2008).

15. A. Mlynek, L. Casali, O. Ford, H. Eixenberger, and A. U. Team, Review of Scientific Instruments 85, 11D408 (2014).

16. R. Kalman, J. Fluids Eng. 82, 35-45 (1960).

17. S. Särkkä, Bayesian Filtering and Smoothing, Cambridge University Press, Cambridge, England, 2013, ISBN 978-1-10761928-9.

18. J. Scargle, Astrophysical Journal 263, 835-853 (1982).

19. G. L. Bretthorst, Bayesian Spectrum Analysis and Parameter Estimation, Springer, Berlin, Germany, 1988, ISBN 3-54096871-7, available online: http://bayes.wustl.edu/glb/book.pdf.

20. K. V. Mardia, and P. E. Jupp, Directional Statistics, John Wiley and Sons, Chichester, England, 2000, ISBN 0-471-95333-4.

21. J. Traa, and P. Smaragdis, IEEE Signal Processing Letters 20, 1257-1260 (2013). 